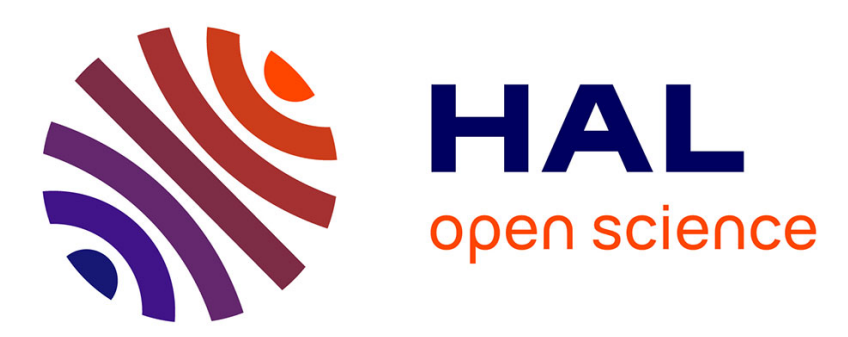

\title{
High-throughput resequencing in the diagnosis of mutations using oligonucleotide resequencing microarrays
}

Christopher Schroeder, Fanny Stutzmann, Bernhard H. F. Weber, Olaf Riess, Michael Bonin

\section{To cite this version:}

Christopher Schroeder, Fanny Stutzmann, Bernhard H. F. Weber, Olaf Riess, Michael Bonin. Highthroughput resequencing in the diagnosis of mutations using oligonucleotide resequencing microarrays. Breast Cancer Research and Treatment, 2009, 122 (1), pp.287-297. 10.1007/s10549-009-0639-z . hal00535415

\section{HAL Id: hal-00535415 \\ https://hal.science/hal-00535415}

Submitted on 11 Nov 2010

HAL is a multi-disciplinary open access archive for the deposit and dissemination of scientific research documents, whether they are published or not. The documents may come from teaching and research institutions in France or abroad, or from public or private research centers.
L'archive ouverte pluridisciplinaire HAL, est destinée au dépôt et à la diffusion de documents scientifiques de niveau recherche, publiés ou non, émanant des établissements d'enseignement et de recherche français ou étrangers, des laboratoires publics ou privés. 


\title{
High-throughput resequencing in the diagnosis of $B R C A 1 / 2$ mutations using oligonucleotide resequencing microarrays
}

\author{
Christopher Schroeder • Fanny Stutzmann • \\ Bernhard H. F. Weber • Olaf Riess • \\ Michael Bonin
}

Received: 27 September 2009/Accepted: 5 November 2009/Published online: 26 November 2009

(C) Springer Science+Business Media, LLC. 2009

\begin{abstract}
Breast cancer is the most frequent form of carcinoma in European females (incidence 65 per 100,000). In about $10 \%$ of all cases, pedigree analysis predicts a hereditary breast-ovarian cancer syndrome (HBOC) to be causative for the disease. Frequently, mutations in two genes, BRCA1 (Chr. 17q21) and BRCA2 (Chr. 13q12), are associated with HBOC. In females, mutations in these genes result in a lifetime risk of $80-85 \%$ for breast cancer and $54 \%$ (BRCA1) or $23 \%$ (BRCA2) for ovarian cancer. Current genetic diagnostic tools for $B R C A 1$ and $B R C A 2$ remain laborious and expensive. Here, we present the first oligonucleotide resequencing microarray covering the complete coding sequence of both genes. In total, 36 previously characterized DNAs were resequenced; all 11 patients with single-nucleotide mutations and, due to a special mutational design, eight patients with heterozygous deletions were detected correctly. In total, 47 different single-nucleotide variants (SNVs) were found. A newly developed software, $\mathrm{SeqC}$, reduced the number of ambiguous calls with the help of a statistical module comparing the acquired data to an online-database. SeqC improved the average call rate to 99\% (GSeq: 97\%) and reduced time and efforts for manual analysis. SeqC confirmed the results obtained by GSeq and found an additional 33 sequences changes representing 14
\end{abstract}

Electronic supplementary material The online version of this article (doi:10.1007/s10549-009-0639-z) contains supplementary material, which is available to authorized users.

C. Schroeder · F. Stutzmann · O. Riess · M. Bonin $(\bowtie)$ Department of Medical Genetics, University of Tübingen, Calwer Str. 7, 72076 Tubingen, Germany

e-mail: Michael.bonin@med.uni-tuebingen.de

B. H. F. Weber

Institute of Human Genetics, University of Regensburg, Franz-

Josef-Stauß-Allee 11, 93053 Regensburg, Germany
SNVs. In total, $945 \mathrm{~kb}$ were screened and the overall turnaround time for each patient took approximately 3 days, including analysis.

Keywords Oligonucleotide resequencing microarray · BRCA1 - BRCA2 - Breast cancer - Ovarian cancer . $\mathrm{HBOC} \cdot \mathrm{HBC} \cdot \mathrm{HOC} \cdot \mathrm{SeqC}$

\section{Introduction}

Breast cancer is the most common form of carcinoma in females in Europe, with an average incidence of 65 per 100,000 individuals [1]. In most patients, the tumor appears sporadic, but it is estimated that at least $10 \%$ involve a genetic predisposition which as a consequence leads multiple manifestation of hereditary breast cancer (HBC) in distinct families [2]. In the early 1990s, BRCAl (Chr. 17q21) and BRCA2 (Chr. 13q12) (Table 1) were identified as high-risk susceptibility genes for autosomal-dominant breast cancer [3, 4]. In addition, follow-up studies were able to link both genes to ovarian cancer (OC) and less frequently to several other types of carcinoma (e.g., melanoma colon, pancreatic, and prostate cancer) [5]. The association of at least $30 \%$ of all HBC to OC led to the term hereditary breast-ovarian cancer syndrome (HBOC). HBOC caused by mutations in BRCA1 or BRCA2 is associated with a lifetime risk of $80-85 \%$ for $\mathrm{BC}$ and $54 \%$ (BRCA1) or 23\% (BRCA2) for OC, respectively [6]. Both BC and $\mathrm{OC}$ are potential life-threatening diseases with an average 5 year-survival rate of $80 \%$ (BC) and $43 \%$ (OC) in Europe [1]. As for every tumor disease prognosis, therapy and survival strongly depend on its stage at diagnosis. Genetic screening tests, especially for patients with a known familial risk of ovarian cancer, are of great value since $71 \%$ of clinically suspicious patients are in stage III or IV of the 
Table 1 Overview on genes associated with a high risk for hereditary breast cancer (HBC) [24]

\begin{tabular}{llll}
\hline Gene & Locus & Ensembl ID & Protein \\
\hline BRCA1 & $17 \mathrm{q} 21$ & ENSG00000012048 & Breast cancer type 1 susceptibility protein \\
BRCA2 & $13 \mathrm{q} 12$ & ENSG00000139618 & Breast cancer type 2 susceptibility protein \\
TP53 & $17 \mathrm{p} 13$ & ENSG00000141510 & Tumor suppressor p53 \\
PTEN & $10 \mathrm{q} 23$ & ENSG00000171862 & Phosphatase tensin homolog on chromosome ten \\
STK11 & $19 \mathrm{p} 13$ & ENSG00000118046 & Serine/threonine-protein kinase 11 \\
CDH1 & $16 \mathrm{q} 22$ & ENSG00000039068 & Epithelial cadherin precursor \\
\hline
\end{tabular}

disease, and, as a consequence, their prognosis is very poor [7]. It is essential to identify these patients and provide genetic counseling to assess their individual disease risk, calculate the probability of mutation-carrier status, and offer genetic analysis. For families with known mutations in $B R C A 1$ or $B R C A 2$, extended preventive screening as well as therapeutic strategies have been established [8].

During the past 18 years, 815 mutations were identified in BRCA1 and 571 in BRCA2 (Human Gene Mutation Database; www.hgmd.cf.ac.uk). However, genetic screening for mutations in the two genes with a total of 50 exons and approximately 17,380 base pairs (including exonintron-boundaries) remains a major challenge. Traditional techniques are direct sequencing, denaturing gradient gel electrophoresis (DGGE), and denaturing high-performance liquid chromatography (dHPLC). Direct sequencing is especially laborious and expensive while DGGE and dHPLC are technically complex and the results that are obtained have to be evaluated by direct sequencing.

Recently developed sequencing tools and resequencing microarrays are more cost- and time-efficient because they allow high-throughput, parallel resequencing of several genes. As proof-of-principle, one of the first oligonucleotide microarrays was designed for a single exon (exon 11) of BRCAl [9]. Over the last 5 years, resequencing oligonucleotide microarrays were established for general mitochondrial mutation analysis [10] and a few diseases like cardiomyopathy [11, 12], intrahepatic cholestasis [13], and retinal degeneration [14]. Our Breast Cancer Microarray is the first oligonucleotide resequencing microarray spanning the complete sequence of BRCA1 and BRCA2. In a first approach, 36 patients were screened for hereditary breast and ovarian cancer. In order to speed up the process, all patients were handled using multiplexes. The SeqC-software (www.jsi-medisys.de) was developed to further accelerate and facilitate analysis of oligonucleotide resequencing microarrays.

\section{Materials and methods}

Array design

The principle of custom resequencing oligonucleotide microarrays uses 25-mer oligonucleotide probes of interest synthesized on an array [15]. Each position of the sequence of interest is represented as the central position of eight different 25-mer oligonucleotides (for each position in the sense and antisense directions four probes with all four different nucleotides). Thus, each sequence is represented in sense and antisense direction with one perfect match and three mismatch nucleotides. This design allows detection of all possible single nucleotide variations. Following the same principle, the most important deletions were designed on the chip with perfectly matched 25 -mer oligonucleotide probes.

The design of the Breast Cancer Microarray was based on the commercially available CustomSEQ array (Affymetrix, Santa Clara). Reference sequences for both genes (BRCA1: GenBank U14680; BRCA2: GenBank U43746) were downloaded from GenBank in FASTA format. The sequence file contained all exons plus $10 \mathrm{bp}$ of each flanking intron. In addition, common deletions and insertions were added to the design (Table 2). The instruction file contained the name of the reference sequence, start and end positions of the fragments, and their fist and last four nucleotides. The final design contained all 50 exons (BRCA1-23, BRCA2-27) plus 61 selected deletions (Table 2). Each array is able to sequence approximately $26,217 \mathrm{bps}$.

Patients and amplification of target sequences

In total, 36 DNAs (33 patients + three reference DNAs) were analyzed. The study was approved by the local ethics committee and all patients gave their informed consent before starting standard diagnostic techniques. In order to validate our Breast Cancer microarray, all 36 DNAs were reanalyzed by means of this extended diagnostic approach. Mutations had been previously characterized by another method (sequencing or dHPLC + sequencing). Five patients were included as controls although obvious disease-causing mutations were not identified by conventional analysis as they carried neutral polymorphisms. A total of 12 patients had disease-causing single nucleotide mutations, while another 16 patients had small deletions of 1-11 base pairs (bp). DNA was isolated from lymphocytes using Roche's Magnapure Compact instrument (Roche, Penzberg, Germany) following the manufacturer's protocol.

Primers were designed with Primer 3 using default settings (http://frodo.wi.mit.edu/cgi-bin/primer3/primer3_www.cgi, 
Table 2 Specific deletions designed on the Breast Cancer Microarray (annotation following BIC-http://research.nhgri.nih.gov)

\begin{tabular}{|c|c|c|c|c|c|}
\hline Gene & Exon & Deletion & Gene & Exon & Deletion \\
\hline \multirow[t]{32}{*}{$B R C A 1$} & 2 & 185delAG & $B R C A 2$ & 2 & IVS2-7delT \\
\hline & 6 & IVS6-2delA & & 2 & L15ins \\
\hline & 11 & D369del & & 3 & N55del \\
\hline & 11 & I482del & & 3 & EL97del \\
\hline & 11 & S616del & & 5 & V145del \\
\hline & 11 & S1140del & & 7 & 802delAT \\
\hline & 11 & Q1281del & & 8 & E215del \\
\hline & 11 & 1675 delA & & 8 & 886delGT \\
\hline & 11 & 2576delC & & 9 & R239ins \\
\hline & 11 & 2594delC & & 9 & 983del4 \\
\hline & 11 & 2800delAA & & 10 & 1983del5 \\
\hline & 11 & 2804delAA & & 10 & 2041insA \\
\hline & 11 & $3450 \mathrm{del} 4$ & & 11 & 5301insA \\
\hline & 11 & 3600del11 & & 11 & T1302del \\
\hline & 11 & 3819del5 & & 11 & S976del \\
\hline & 11 & 3875 del4 & & 11 & K1286del \\
\hline & 11 & S1297del & & 11 & E1382del \\
\hline & 11 & 4154delA & & 11 & 6503delTT \\
\hline & 11 & 4184del4 & & 11 & 6174delT \\
\hline & 17 & V1688del & & 11 & 5950delCT \\
\hline & 17 & H1673del & & 11 & 5804del4 \\
\hline & 18 & A1693del & & 11 & 5578delAA \\
\hline & 19 & Q1721del & & 11 & 4706delAAAG \\
\hline & 20 & G1738del & & 11 & 4075delGT \\
\hline & & & & 11 & 3036del4 \\
\hline & & & & 11 & 2157delG \\
\hline & & & & 14 & 7297delCT \\
\hline & & & & 15 & K2498del \\
\hline & & & & 18 & K2750del \\
\hline & & & & 18 & $8525 \mathrm{delC}$ \\
\hline & & & & 22 & 9132delC \\
\hline & & & & 25 & 9663delGT \\
\hline
\end{tabular}

Supplementary Table S1). Three PCR-approaches were used to generate all 50 amplicons ranging from 0.190 to $5.12 \mathrm{kbp}$ (PCR-protocols Supplementary Table S1). Nine multiplexes with four to five amplicons each (Table 3), five single-PCRs, and three long-range PCRs were established to amplify the coding regions of both genes. A total of $1.0 \mu \mathrm{g}$ DNA per patient was needed for amplification. The quality of all PCR products was determined using an Agilent Bioanalyzer 2100 (multiplexes) and gel electrophoresis (single-plex).

Chip processing and analysis

According to the protocols provided by Affymetrix, all amplicons were pooled and DNA was purified using a
Table 3 PCR-design for multiplex-reactions

\begin{tabular}{lll}
\hline Multiplex & Gene & Exon \\
\hline 1 & BRCA1 & $1,4,9,14$ \\
& BRCA2 & 21 \\
2 & $B R C A 1$ & $3,8,15$ \\
& BRCA2 & 13 \\
3 & $B R C A 1$ & $5,16,17,23$ \\
4 & $B R C A 1$ & 13,18 \\
& $B R C A 2$ & 1,3 \\
5 & $B R C A 1$ & $11,12,20$ \\
& $B R C A 2$ & 4,7 \\
6 & $B R C A 1$ & 21,22 \\
& $B R C A 2$ & $2,9,16$ \\
7 & $B R C A 2$ & $12,14,18,23$ \\
8 & $B R C A 2$ & $17,22,24,25$ \\
9 & $B R C A 1$ & 19 \\
& $B R C A 2$ & $5,6,8,20,26$ \\
\hline
\end{tabular}

QIAquick PCR purification Kit (Qiagen, Hilden). The following steps (fragmentation, labeling, and hybridization) were performed as recommended by the manufacturer (GeneChip CustomSeq Resequencing Array Protocol Version 5.0, Affymetrix). All arrays were stained, washed using automated fluidic stations, and scanned with a GeneChip 3000 Scanner (Affymetrix, Santa Clara). Affymetrix GCOS Vers. 1.4 and GSEQ 4.0 Software (default settings) were used to process raw data and analyze the nucleotide sequences. SeqC Vers. 3.2.1.5 (JSI-medisys, www.jsi-medisys.de), was used to re-analyze the acquired datasets. SeqC is a module of the SeqPilot software and was originally used to analyze sequencing electropherograms from capillary sequencing. For further analysis, the generated data were compared with the Breast Cancer Mutation Database (http://research.nhgri.nih.gov/pro jects/bic/) and Human Genome Mutation Database (www. hgmd.cf.ac.uk/). In cases of contradicting database entries, the Breast Cancer Mutation Database annotation was finally used.

\section{Results}

The developed Breast Cancer Microarray was evaluated with 36 previously characterized DNAs (Table 4). In total, about $945 \mathrm{kbp}(26,217 \mathrm{bp}$ per patient) were amplified, processed, and analyzed. Using the newly developed software, SeqC, an average call rate of $99 \%$ was reached (GSeq-97\%). The call rate improved with the rising number of chip experiments. 
Table 4 Overview of all identified sequence chances including mutations, singlenucleotide variants, chip call rate and total amount of ambiguous calls (Ns) for each patient
Mutations in brackets were not designed on the chip. Asteriskmarked DNAs were amongst the first microarrays and are therefore of lower quality. Although these arrays were included to demonstrate the noise reduction of $\mathrm{SeqC}$ in comparison to GSeq

\begin{tabular}{|c|c|c|c|c|c|c|}
\hline DNA & Mutation (cDNA) & $\begin{array}{l}\text { Call rate Chip } \\
\text { SeqC/GSeq }\end{array}$ & $\begin{array}{l}\text { SNVs } \\
\text { SeqC/GSeq }\end{array}$ & $\begin{array}{l}\text { Polymorphisms } \\
\text { (SeqC) }\end{array}$ & $\begin{array}{l}\text { Mutation } \\
\text { (SeqC) }\end{array}$ & $\begin{array}{l}\text { Ns } \\
\text { (SeqC) }\end{array}$ \\
\hline 1 & - & $99 \% / 99 \%$ & $20 / 115$ & 17 & - & 3 \\
\hline 2 & - & $99 \% / 98 \%$ & $27 / 504$ & 12 & - & 15 \\
\hline 3 & - & $99 \% / 98 \%$ & $27 / 440$ & 14 & - & 13 \\
\hline 4 & - & $99 \% / 97 \%$ & $28 / 399$ & 14 & - & 14 \\
\hline 5 & - & $99 \% / 96 \%$ & $159 / 441$ & 15 & - & $*$ \\
\hline 6 & - & $99 \% / 97 \%$ & $20 / 297$ & 17 & - & 3 \\
\hline 7 & - & $99 \% / 98 \%$ & $17 / 100$ & 13 & - & 4 \\
\hline 8 & - & $99 \% / 98 \%$ & $19 / 161$ & 14 & - & 5 \\
\hline 9 & (BRCA2 8358delT) & $99 \% / 98 \%$ & $23 / 205$ & 11 & - & 12 \\
\hline 10 & (BRCA2 1727delG) & $99 \% / 98 \%$ & $33 / 165$ & 6 & - & 27 \\
\hline 11 & (BRCA2 3972delTGAG) & $99 \% / 97 \%$ & $18 / 176$ & 13 & - & 5 \\
\hline 12 & (BRCA2 1257delA) & $99 \% / 97 \%$ & $16 / 487$ & 6 & - & 10 \\
\hline 13 & (BRCA2 6503delTT) & $99 \% / 96 \%$ & $240 / 3062$ & 5 & - & $*$ \\
\hline 14 & (BRCA1 3040insT) & $99 \% / 97 \%$ & $63 / 335$ & 13 & - & 50 \\
\hline 15 & (BRCA1 3874delTGTC) & $99 \% / 96 \%$ & $172 / 468$ & 14 & - & $*$ \\
\hline 16 & (BRCA1 5382insC) & $99 \% / 97 \%$ & $88 / 293$ & 14 & - & 74 \\
\hline 17 & $B R C A 1 \mathrm{~T}>\mathrm{G} 5228$ & $99 \% / 96 \%$ & $166 / 886$ & 4 & 1 & $*$ \\
\hline 18 & $B R C A 1 \mathrm{C}>\mathrm{T} 297$ & $99 \% / 97 \%$ & $38 / 209$ & 13 & 1 & 24 \\
\hline 19 & BRCA1 C > G 4808 & $99 \% / 94 \%$ & $247 / 2676$ & 5 & 1 & $*$ \\
\hline 20 & $B R C A 1 \mathrm{G}>\mathrm{A} 5629$ & $99 \% / 95 \%$ & $219 / 629$ & 14 & 1 & $*$ \\
\hline 21 & $B R C A 1$ IVS $21 \mathrm{G}>\mathrm{A}-1$ & $99 \% / 98 \%$ & $24 / 174$ & 6 & 1 & 17 \\
\hline 22 & $B R C A 1 \mathrm{C}>\mathrm{T} 297$ & $99 \% / 97 \%$ & $45 / 442$ & 15 & 1 & 29 \\
\hline 23 & BRCA1 C > T 4302 & $99 \% / 98 \%$ & $29 / 219$ & 14 & 1 & 14 \\
\hline 24 & $B R C A 1 \mathrm{C}>\mathrm{A} 2428$ & $99 \% / 99 \%$ & $10 / 82$ & 6 & 1 & 3 \\
\hline 25 & $B R C A 1 \mathrm{G}>\mathrm{A} 4304$ & $99 \% / 98 \%$ & $15 / 100$ & 12 & 1 & 2 \\
\hline 26 & $B R C A 1 \mathrm{C}>\mathrm{T} 4341$ & $99 \% / 98 \%$ & $19 / 136$ & 15 & 1 & 3 \\
\hline 27 & BRCA1 C > G 4808 & $99 \% / 98 \%$ & $9 / 94$ & 6 & 1 & 2 \\
\hline 28 & $B R C A 2 \mathrm{~A}>\mathrm{T} 6265$ & $99 \% / 98 \%$ & $14 / 182$ & 10 & 1 & 3 \\
\hline 29 & BRCA1 4184delTCAA & $99 \% / 97 \%$ & $33 / 223$ & 14 & - & 19 \\
\hline 30 & BRCA1 4184delTCAA & $99 \% / 96 \%$ & $94 / 362$ & 11 & - & $*$ \\
\hline 31 & BRCA2 4075delGT & $99 \% / 98 \%$ & $26 / 171$ & 5 & - & 21 \\
\hline 32 & BRCA1 3874delTGTC & $99 \% / 98 \%$ & $28 / 200$ & 14 & - & 14 \\
\hline 33 & $\begin{array}{l}\text { BRCA1 } \\
\quad \text { 3600delGAAGATACTAG }\end{array}$ & $99 \% / 98 \%$ & $38 / 233$ & 15 & - & 23 \\
\hline 34 & $\begin{array}{l}\text { BRCA1 } \\
\text { 3600delGAAGATACTAG }\end{array}$ & $99 \% / 98 \%$ & $18 / 157$ & 11 & - & 7 \\
\hline 35 & BRCA1 4184delTCAA & $99 \% / 98 \%$ & $6 / 95$ & 4 & - & 2 \\
\hline 36 & BRCA2 3036delACAA & $99 \% / 98 \%$ & $12 / 102$ & 8 & - & 4 \\
\hline
\end{tabular}

Table 3. As a consequence, for each patient, 17 PCRs were necessary and, overall, 612 PCR-reactions were performed in this study. Due to the use of multiplexes, the total number of PCRs and duration of pipetting was reduced to about one-third, saving chemicals and hands-on-time.

New software

Data analysis was managed as a two-step process. In the first run, all patients were analyzed using GSeq, while in 
the second run, they were re-analyzed using SeqC. Samples marked with asterisks (Table 4) were excluded from the following statistical analysis. GSeq reached an average call rate (CR) of $97 \%$. About 212 loci per patient were Ns, since standard software parameters were not able to resolve the designated bases. These Ns must be evaluated manually and the corresponding fluorescence-signals have to be screened for sequence changes. In our patients and with GSeq, $75 \mathrm{Ns}$ in BRCA1 and $137 \mathrm{Ns}$ in BRCA2 had to be evaluated manually. On the basis of the GSeq result files, SeqC was able to generate an average CR of $99 \%$ with its own basecaller and consequently reduced the overall number of Ns. Using SeqC, only 15 different loci for both genes (7\% of Ns in GSeq) were found and had to be evaluated manually. The average patient had $5 \mathrm{Ns}$ in $B R C A 1$ and 9 Ns in BRCA2. Due to the length of BRCA1 Exon 10 and BRCA2 Exon $10+11$, both SeqC and GSeq found most of the ambiguous calls in these exons. The average of $15 \mathrm{Ns}$ plus an average of 12 polymorphisms per patient led to approximately $27 \mathrm{SNVs}$ per patient.

Analysis with GSeq and evaluating ambiguous calls by comparing fluorescence intensities is especially laborious. In contrast to GSeq, SeqC offers a simple table with all Ns. Each $\mathrm{N}$ is directly linked to the corresponding position in the electropherogram and to a statistical module. The electropherogram helps to control the hybridization quality at that specific locus plus the nucleotides close to it. The statistical module compares each selected base with already archived chips, showing the medium relative intensity and the standard deviation. In order to maintain comparable chips, each chip had to be assigned to a PCR arrangement and only chips of the same arrangement were compared. In order to further facilitate analysis of oligonucleotide resequencing microarrays, $\mathrm{SeqC}$ was able to compare sequence changes with an online-database (e.g., Ensembl, www.ensembl.org) and use the official nomenclature for base changes as well as the amino acid changes.

Due to the different underlying reference sequences in SeqC and in GSeq, SeqC found three additional SNPs (rs206075, rs206076, and rs169547) that were homozygous in all patients and one SNP (rs543304) was detected in the complementary genotype. The newly discovered homozygous SNPs and rs543304 were not considered in the comparison of GSeq and SeqC. In total, SeqC was able to confirm the results of GSeq and added another 35 changes of 16 SNVs which would have been missed using GSeq alone (Fig. 1). Of these different $16 \mathrm{SNVs}$, three were only detected in SeqC with one occurrence. One result of this study is the confirmation that the different approach used by $\mathrm{SeqC}$ proved to be very efficient and reduced the duration of analysis to about one-tenth of the time.
Analysis of $B R C A 1 / 2$ oligonucleotide resequencing microarrays

All 12 out of 12 patients with single-base mutations were confirmed (Fig. 2) by our re-sequencing microarray analysis, and the special design to detect known mutations recognized all eight theoretically detectable deletions (Fig. 3). In total, 48 different single-nucleotide variants (SNVs) with 415 occurrences were found among all the 36 DNAs using SeqC with standard software parameters (Table 4). All sequence variants were compared to the breast cancer mutation database and the human gene mutation database. The clinical relevance of all mutations (single-nucleotide and deletions) was confirmed (Table 5). The clinical relevance of $15 \mathrm{SNVs}$ was marked as "unknown" in at least one of the two databases. Two occurrences of variants exclusively detected with SeqC were of unknown clinical importance: Patient 6-rs4987046 and rs28897762. Three SNVs (rs28897706, rs144848, and rs11571833) led to completely contradicting results in both online databases (Table 5). These three polymorphisms were designated as recommended by the Breast Cancer Mutation Database as being of "no clinical relevance". In total, 13 different SNVs marked as "unknown clinical relevance" were detected following the breast cancer mutation database. Two of them (rs1801499 and rs28897762) result in a synonymous amino acid change and are therefore not likely to be deleterious. The remaining 11 SNVs of "unknown clinical relevance" may contribute to HBOC. In total, 19 SNPs did not seem to be related to HBOC and another five SNPs could not be found. Two SNVs (BRCA1 S784S, BRCA2 C> T-143) were found neither in Ensembl (www.ensembl.org) nor both mutation databases. Due to the synonymous amino acid change it is likely that S784S is a new, neutral polymorphism described in this study. All eight detectable heterozygous deletions were properly detected manually (Table 4). In contrast to SeqC, GSeq is not able to evaluate the special perfect match design for mutations. Although all perfect match deletion sequences (Table 2) were analyzed manually. An elevated relative fluorescence-signal indicates a deletion in a patient. Figure 3 illustrates an example of manual deletion detection with patient 30 .

Accuracy, specificity, and sensitivity of CustomSeq resequencing arrays have been proven by correctly recognizing 25 occurrences of previously analyzed SNVs within our positive controls. In order to confirm reproducibility, a total of six chips were amplified and hybridized at least twice with equal results using SeqC. Detecting hemizygous deletions remained laborious; we were not able to safely detect hemizygous deletions that were not designed on the chip. 
Fig. 1 Illustration of two SNPs dedicated as Ns by GSeq but automatically detected using SeqC. The electropherogram shown in this figure is used for an additional manual evaluation. Heterozygous SNVs are presented as elevated fluorescence-signals for two bases at the same time (APatient 32), whereas homozygous SNVs are characterized by a single-base, normal fluorescence-signal at the center position and a lack of fluorescence-signal around the center position due to a mismatch (B-Patient 11)

\section{A rs1799966}

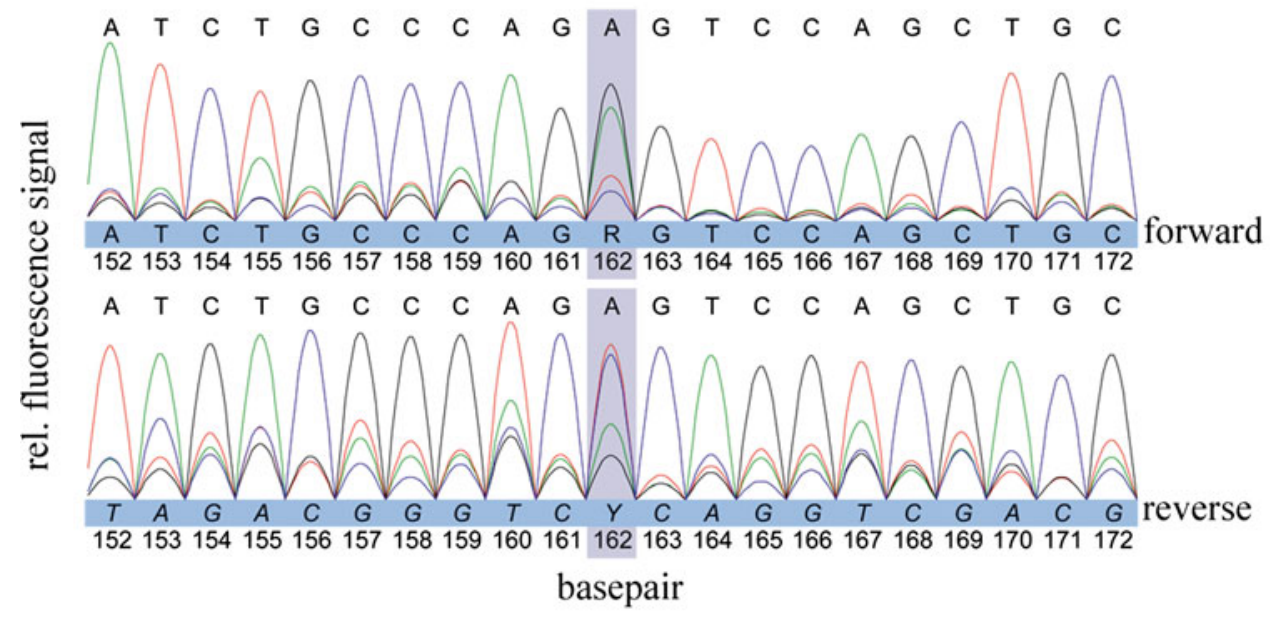

B rs1799955

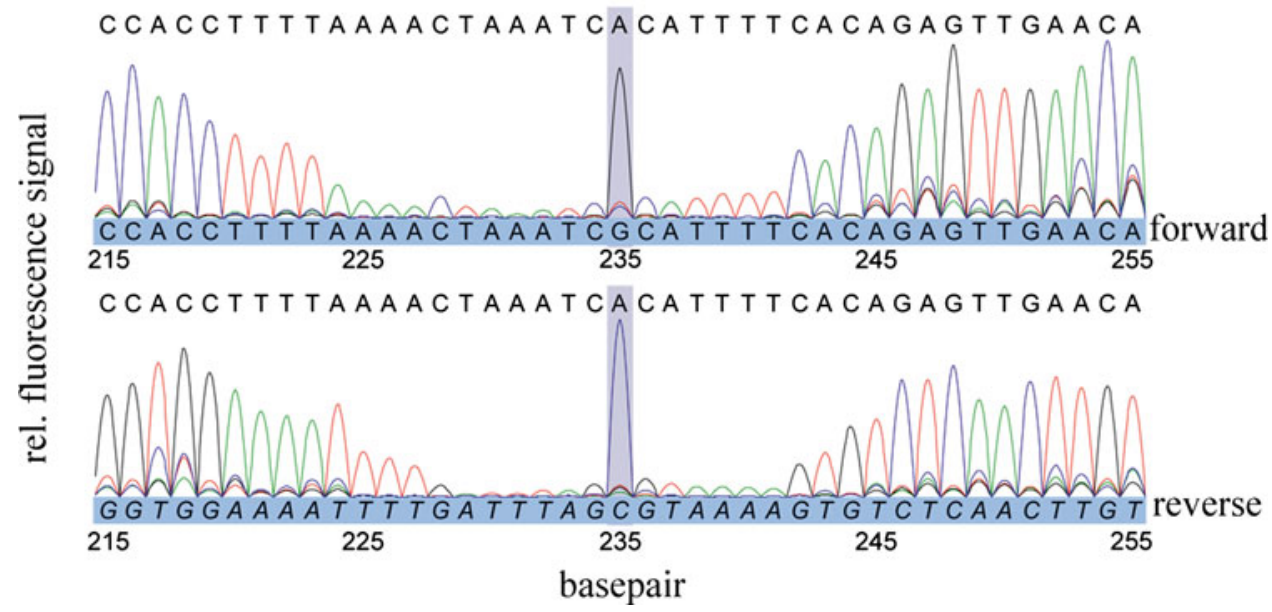

Using SeqC and multiplexes, each patient took approximately 3 days, including analysis. PCR amplification and pooling took place on the first day. On the second day, the sample was processed and hybridized to the chip, then on the third day, staining, scanning, and analysis were carried out. All ambiguous calls were evaluated manually and therefore a very dense map with high informative value for all SNVs was generated.

\section{Discussion}

Microarray technology is an important tool to analyze cancer genomes and to understand the causes of cancer [16]. Sequencing microarrays have been appreciated as a valid tool for parallel and accurate resequencing of genes. In the last few years, the total number of different custom oligonucleotide resequencing microarrays has increased and this technique has been successfully applied in mitochondrial mutation analysis [10], cardiomyopathy
[11, 12], intrahepatic cholestasis [13], and retinal degeneration [14]. This study focused on two genes, BRCA1 and BRCA2, that are causative for HBOC [5]. Altogether, 33 positive controls and three standard DNAs with a total of $945 \mathrm{~kb}$ were analyzed and all previously known SNVs were detected with high accuracy (100\%, 25/25). In addition to this, we were able to confirm high reproducibility by repeating different experiments. Previous studies showed comparable results concerning accuracy (99.9-100\%) and reproducibility [10-14]. These studies also reported call rates ranging from 93.5 to $97.6 \%$.

A new program, SeqC, was used in our study to analyze the data. With the help of this tool, the average call rate could be raised from $97 \%$ using the standard analysis-tool GSeq to $99 \%$. While comparing the results of GSeq and $\mathrm{SeqC}$, no loss of information was detected and known single nucleotide mutations could be evaluated in both programs. Although most of the SNVs were detected with both GSeq and SeqC, we were able to find 35 additional 
Fig. 2 Detection of mutations. The detection of mutations corresponds to the detection of SNVs. This figure shows two mutations that were automatically detected by SeqC as well as GSeq. As part of the analysis, SeqC indicates the correct cDNA position and amino acid change in the protein. Both mutations (A-Patient 18, B-Patient 19) shown in this image lead to a frame shift and result in early termination of the protein

\section{A $B R C A 1 \mathrm{Q} 60 \mathrm{X}$}

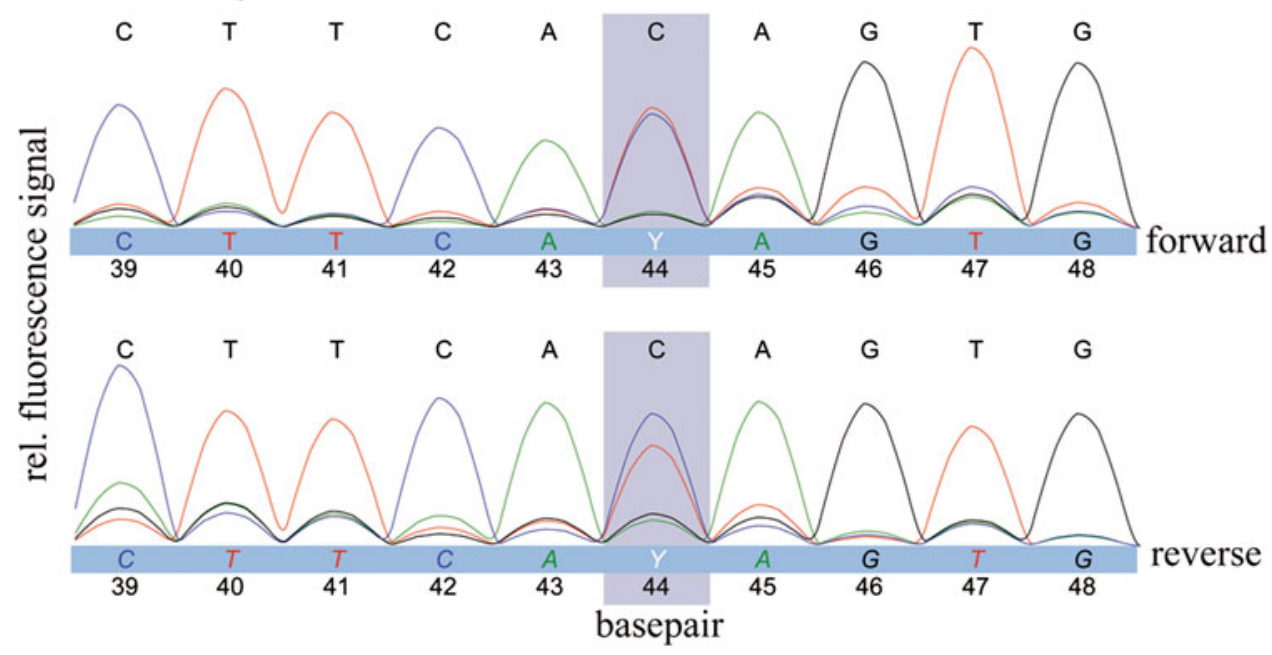

\section{B $B R C A I \mathrm{Y} 1563 \mathrm{X}$}

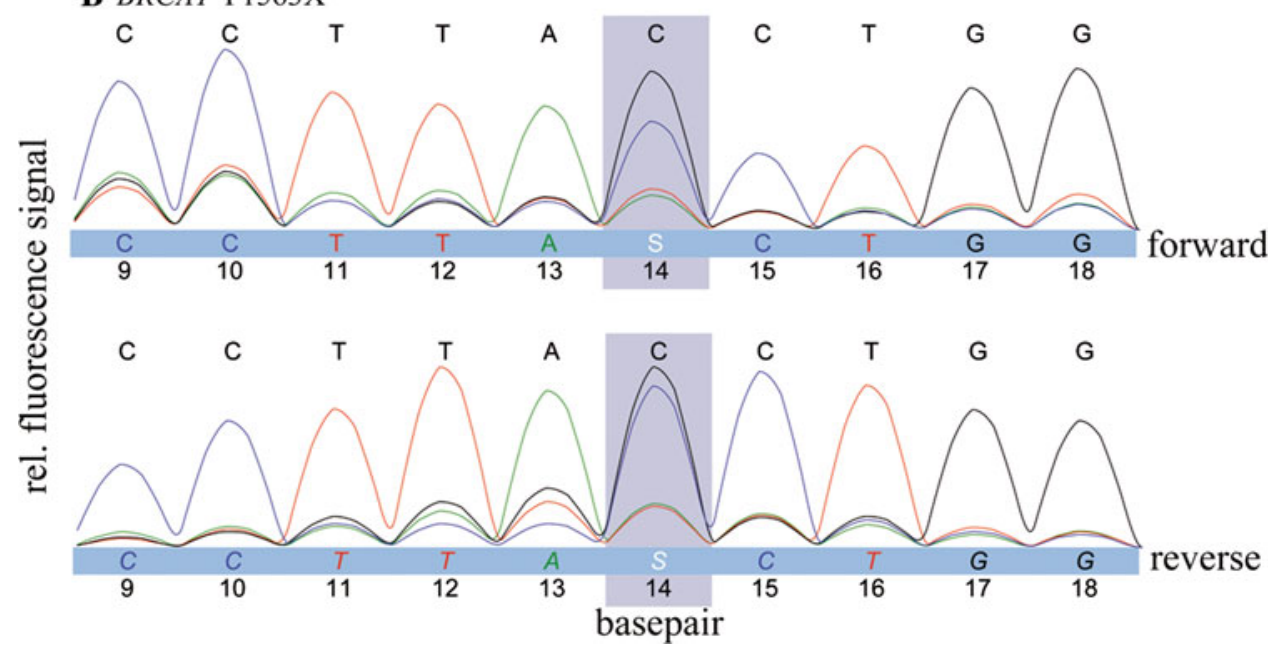

occurrences of 16 SNVs with SeqC (Fig. 1), and three SNVs were exclusively found in SeqC.

Additional features of SeqC facilitated the analysis of the data. In the end, an average of 15 ambiguous calls (Ns) had to be evaluated manually for both genes. A statistical module compared each nucleotide with already archived arrays and visualized the average fluorescence intensity and standard deviation at each position. An electropherogram indicated current signal intensities and their course. The most time-consuming factor, data analysis, was effectively reduced, saving hands-on time and money. In addition, we successfully established multiplexes (Table 3) and were able to further reduce time of pipetting and use of chemicals.

Two approaches for detection of deletions and insertions are theoretically possible. One is to design special perfect match (PM) oligonucleotides for deleted or inserted sequences [17, 18]. These oligonucleotides led to an increase in hybridization signal for every patient with that specific deletion compared with wild type sequence. This approach has the advantage of both detecting the presence and identifying the sequence change, but is limited by the size of the microarray and the total number of mutationspecific oligonucleotides. The second possibility is to detect sequence changes based on a loss of hybridization signal within the wild type sequence. Consequently, the sequence variation is only detected but then needs further identification (for example by sequencing). This approach has been described in the literature to be effective with deletions of more than $50 \mathrm{bp}$ [16]. Since insertions and deletions are important in BRCA1 and BRCA2, perfectly match oligonucleotides were designed on the chip for the most important deletions and insertions (Table 2). With the help of these oligonucleotides, all eight detectable heterozygous deletions were detected with respect to the hybridization signal (Figure 3), however, de novo detection of heterozygous deletions due to the loss of hybridization signal remains difficult. Large genomic heterozygous 
Fig. 3 Detection of deletions. Most important deletions were designed as perfect match probes on the oligonucleotide resequencing microarray. Samples with that special deletion resulted in a perfectedmatch and normal hybridization signal. DNA without the mutation is theoretically not able to hybridize to the deletionspecific sequence and results in little or no signal. The signal intensity of heterozygous mutation carriers will decrease but is still maintained at significantly elevated level. Since software algorithms cannot consider perfect match deletion sequences, each probe has to be checked manually for all patients. For illustration purposes, the quotient of relative signal-intensities mutated/wildtype for a deletionspecific sequence (BRCA1 4184delTCAA) was plotted on the $y$-axis. The data shown is based on Patient 30,

heterozygous for $B R C A 1$ 4184delTCAA, and one control (patient 3). The increase of signal in relation to the wild type sequence at the central position indicates the presence of the deletion
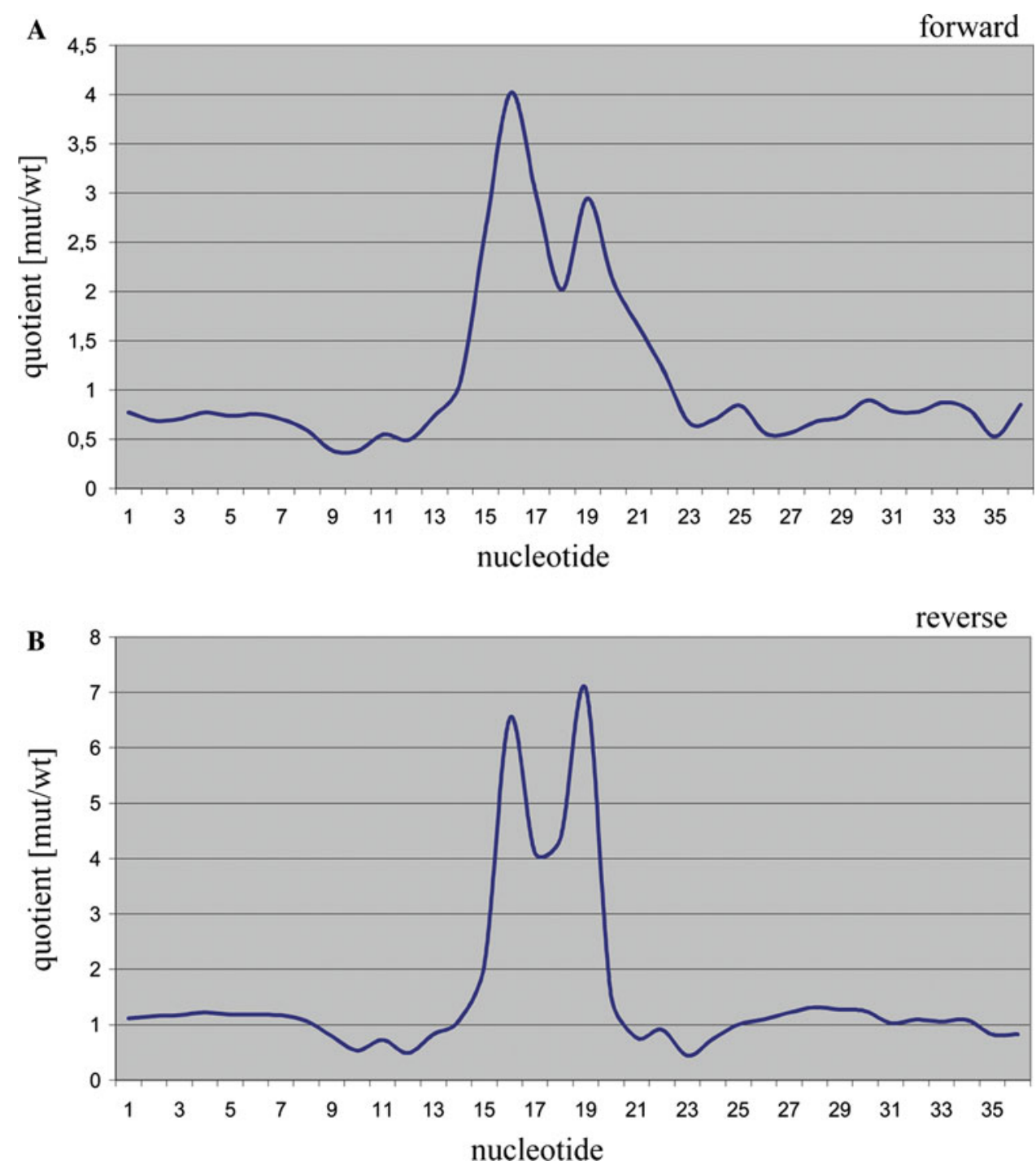

deletions, which may occur in BRCA1, can theoretically be identified using specially designed oligonucleotide probes at the end or the beginning of a specific deletion. Since, both standard Sanger-Sequencing and olignoculeotide resequencing microarrays have difficulties in detecting large heterozygous deletions, a second (screening) method such as multiplex ligation-dependent probe amplification may be used before (re)sequencing [19]. In contrast to heterozygous deletions, de novo homozygous deletions can be detected easily by the total loss of hybridization signal.

Sequence changes in BRCA1 and BRCA2 can be subdivided by their clinical relevance (yes/no/unknown, see http://research.nhgri.nih.gov/projects/bic/). For sequence changes with questionable relationships to $\mathrm{HBOC}$, recent studies have tried to develop and apply models to predict their clinical relevance [20-22]. Although all examined patients in this study were previously characterized, screening for mutations ended whenever a deleterious sequence change was found. With the help of the Breast
Cancer Microarray, we were able to construct a complete map of single-nucleotide variations for each patient. With this additional information, a total of 19 SNPs that were not thought to be relevant in HBOC and another five SNPs that did not appear in the BIC database could be identified. Thirteen SNVs of unknown relevance in regards to HBOC were found and further studies are needed to prove their clinical relevance.

The results of this study, as well as those in the literature with oligonucleotide resequencing microarrays, are comparable to sequencing techniques like the standard Sanger sequencing and so-called second-generation sequencing tools [23]. Oligonucleotide resequencing microarrays are equal in sensitivity and specificity to both techniques. An advantage of oligonucleotide microarrays is its cost-effectiveness; about $70 \%$ less compared to standard Sanger sequencing. For second-generation sequencing tools, further evaluation is needed and costs for routine usage must decrease prior to broad application. 
Table 5 Single-nucleotide variants (SNV) found among all screened DNAs and their clinical importance following HGMD and BIC $(-=$ not listed; ? = unknown/variant of unkown significance; yes clinical important; no clinical unimportant, Mut mutation)

\begin{tabular}{|c|c|c|c|c|c|c|c|}
\hline Gene & Exon & cDNA & Amino Acid & Accession & Frequency & HGMD & $\mathrm{BIC}$ \\
\hline BRCAl & 5 & C > T 297 & Q60X & Mut. & 2 & - & yes \\
\hline BRCA1 & 5 & $\mathrm{~T}>\mathrm{G} 300$ & C61G & Mut. rs28897672 & 1 & yes & yes \\
\hline BRCAl & 5 & IVS $5+3 \mathrm{~A}>\mathrm{G}$ & $3^{\prime}$ UTR & Mut. & 1 & yes & $?$ \\
\hline$B R C A 1$ & 11 & $\mathrm{~A}>\mathrm{G} 1186$ & Q356R & rs 1799950 & 4 & $?$ & $?$ \\
\hline BRCAl & 11 & G > A 2196 & D693 N & Polymorphism & 2 & $?$ & no \\
\hline$B R C A 1$ & 11 & $\mathrm{C}>\mathrm{T} 2201$ & S694S & rs1799949 & 21 & - & no \\
\hline BRCA1 & 11 & C > A 2428 & S770X & Mut. & 1 & yes & yes \\
\hline$B R C A 1$ & 11 & $\mathrm{~T}>\mathrm{C} 2430$ & L771L & rs16940 & 25 & no & no \\
\hline BRCA1 & 11 & $\mathrm{G}>$ A 2471 & S784S & & 1 & - & - \\
\hline BRCAl & 11 & $\mathrm{C}>\mathrm{T} 2731$ & P871L & rs799917 & 24 & - & no \\
\hline$B R C A 1$ & 11 & A $>$ G 3232 & E1038G & rs16941 & 25 & $?$ & no \\
\hline BRCAl & 11 & $\mathrm{G}>$ A 3238 & S1040 N & rs4986852 & 1 & $?$ & $?$ \\
\hline$B R C A 1$ & 11 & A > G 3667 & K1183R & rs16942 & 25 & - & no \\
\hline$B R C A 1$ & 11 & $\mathrm{~A}>\mathrm{G} 4158$ & R1347G & rs 28897689 & 2 & $?$ & $?$ \\
\hline BRCAl & 12 & $\mathrm{C}>\mathrm{T} 4302$ & Q1395X & Mut. & 1 & yes & yes \\
\hline BRCA1 & 12 & $\mathrm{G}>$ A 4304 & Q1395Q & Mut. & 1 & - & yes \\
\hline BRCA1 & 13 & $\mathrm{C}>\mathrm{T} 4341$ & Q1408X & Mut. & 1 & yes & yes \\
\hline BRCAl & 13 & $\mathrm{~T}>\mathrm{C} 4427$ & S1436S & rs1060915 & 25 & - & no \\
\hline BRCAl & 16 & $C>$ G 4808 & Y1563X & Mut. & 2 & yes & yes \\
\hline$B R C A 1$ & 16 & $\mathrm{~A}>\mathrm{G} 4956$ & S1613G & rs1799966 & 25 & - & no \\
\hline$B R C A 1$ & 16 & $\mathrm{G}>\mathrm{A} 5075$ & M1652I & rs1799967 & 2 & $?$ & $?$ \\
\hline BRCA1 & 18 & $\mathrm{~T}>$ G 5228 & Y1703X & Mut. & 1 & - & yes \\
\hline BRCAl & 21 & IVS $21 \mathrm{G}>\mathrm{A}-1$ & $5^{\prime}$ UTR & Mut. & 1 & - & yes \\
\hline BRCA1 & 24 & $\mathrm{G}>\mathrm{A} 5629$ & W1837X & Mut. & 1 & yes & yes \\
\hline BRCA2 & 1 & $\mathrm{C}>\mathrm{T}-143$ & $5^{\prime}$ UTR & & 1 & - & - \\
\hline BRCA2 & 1 & $A>G-52$ & $5^{\prime}$ UTR & rs206118 & 13 & - & - \\
\hline BRCA2 & 2 & $\mathrm{G}>\mathrm{A}-26$ & $5^{\prime}$ UTR & rs1799943 & 17 & - & - \\
\hline BRCA2 & 3 & $\mathrm{~A}>\mathrm{G} 125$ & Y42C & rs4987046 & 1 & $?$ & $?$ \\
\hline BRCA2 & 10 & A > C 1093 & $\mathrm{~N} 289 \mathrm{H}$ & rs766173 & 1 & - & no \\
\hline BRCA2 & 10 & C > A 1206 & S326R & rs 28897706 & 1 & yes & no \\
\hline BRCA2 & 10 & C > A 1342 & H372 N & rs144848 & 14 & yes & no \\
\hline BRCA2 & 10 & A > G 1593 & S455S & rs1801439 & 1 & - & no \\
\hline BRCA2 & 11 & $\mathrm{~T}>\mathrm{C} 2457$ & $\mathrm{H} 743 \mathrm{H}$ & rs1801499 & 1 & - & $?$ \\
\hline BRCA2 & 11 & A > G 3199 & N991D & rs1799944 & 1 & - & no \\
\hline BRCA2 & 11 & A > G 3624 & K1132 K & rs1801406 & 20 & - & no \\
\hline BRCA2 & 11 & $\mathrm{~T}>\mathrm{C} 4035$ & V1269 V & rs543304 & 16 & - & no \\
\hline BRCA2 & 11 & A > G 4791 & L1521L & rs206075 & 36 & - & no \\
\hline$B R C A 2$ & 11 & $\mathrm{C}>\mathrm{T} 5427$ & S1733S & rs28897734 & 2 & - & no \\
\hline BRCA2 & 11 & $\mathrm{~T}>\mathrm{C} 5972$ & M1915I & rs4987117 & 2 & $?$ & $?$ \\
\hline BRCA2 & 11 & $\mathrm{~A}>\mathrm{T} 6265$ & K2013X & Mut. & 1 & yes & yes \\
\hline BRCA2 & 11 & C > T 6328 & R2034C & rs1799954 & 1 & $?$ & $?$ \\
\hline$B R C A 2$ & 11 & $\mathrm{G}>\mathrm{C} 6741$ & V2171 V & rs206076 & 36 & - & no \\
\hline$B R C A 2$ & 14 & $\mathrm{G}>\mathrm{A}-26$ & $5^{\prime}$ UTR & rs1799943 & 1 & - & - \\
\hline BRCA2 & 14 & $\mathrm{~A}>\mathrm{G} 7470$ & $\mathrm{~S} 2414 \mathrm{~S}$ & rs1799955 & 15 & - & no \\
\hline BRCA2 & 14 & $\mathrm{~T}>\mathrm{C} 7625$ & V2466A & rs169547 & 36 & no & no \\
\hline BRCA2 & 22 & $\mathrm{G}>\mathrm{T} 9078$ & K2950 N & rs28897754 & 1 & yes & $?$ \\
\hline
\end{tabular}


Table 5 continued

\begin{tabular}{|c|c|c|c|c|c|c|c|}
\hline Gene & Exon & cDNA & Amino Acid & Accession & Frequency & HGMD & $\mathrm{BIC}$ \\
\hline BRCA2 & 27 & $\mathrm{G}>\mathrm{A} 10110$ & R3370R & rs28897762 & 1 & - & $?$ \\
\hline BRCA2 & 27 & $\mathrm{~A}>\mathrm{T} 10204$ & K3326X & rs11571833 & 1 & yes & no \\
\hline
\end{tabular}

Known deletions are marked in gray

Mutations in BRCA1 and BRCA2 are the cause for the hereditary breast-ovarian cancer syndrome (HBOC). HBOC is the underlying syndrome of about one-third of all hereditary breast cancer cases [5] and accounts for $15 \%$ of all cases of ovarian cancer, the majority of hereditary ovarian cancers [7]. It has been well established that patients with a family risk of breast or ovarian cancer need to be included in special, extended screening programs, which are based on general screening techniques like monthly breast self examination, clinical breast examination (every six months), and imaging (annual mammography or magnet resonance imaging), which will help to detect and treat breast cancer at the early stages [24]. Despite this secondary prevention, all patients with a family risk for HBOC should undergo genetic counseling and genetic testing [24]. For patients with known HBOC, effective broad-spectrum prophylactic treatment can be offered, ranging from chemopreventive medication to prophylactic surgery (bilateral mastectomy, salpingooophorectomy). Primary prevention activities should also be carried out with a multidisciplinary team with geneticists and psycho-oncologists [24]. Since an effective cancer screening and prophylactic treatment is possible, appropriate risk assessment and genetic testing is essential. At this point, the high-throughput oligonucleotide resequencing microarray presented in this study, spanning the complete sequence of BRCA1 and BRCA2, has proven to be safe, fast, and affordable. Future applications of this tool might comprise assessment of modifying sequence changes and resequencing of other genes known to contribute to breast cancer, e.g., TP53, PTEN, STK11, and CDH1 [25].

\section{Conclusions}

This study successfully establishes the first high-throughput oligonucleotide resequencing microarray for the total sequence of BRCA1 and BRCA2 to supply a safe, fast, and affordable tool to diagnose SNVs, homozygous, and selected heterozygous deletions in patients at high risk for HBOC. In addition, a new software tool, SeqC, facilitated and fastened the previous laborious analysis of chip data by reducing the absolute number of Ns and clearly arranging the remaining data with the help of online databases.
Acknowledgments We thank JSI medical systems and especially Joachim Strub and Dr. Volker Horejschi for assistance during data analysis. The analysis of BRCA patients was supported by a multicenter grant from the Deutsche Krebshilfe, Bonn, Germany.

\section{References}

1. Karim-Kos HE, de Vries E, Soerjomataram I, Lemmens V, Siesling S, Coebergh JW (2008) Recent trends of cancer in Europe: a combined approach of incidence, survival and mortality for 17 cancer sites since the 1990s. Eur J Cancer 44:1345-1389

2. McPherson K, Steel CM, Dixon JM (2000) ABC of breast diseases. Breast cancer-epidemiology, risk factors, and genetics. BMJ 321:624-628

3. Hall JM, Lee MK, Newman B, Morrow JE, Anderson LA, Huey B, King MC (1990) Linkage of early-onset familial breast cancer to chromosome 17q21. Science 250:1684-1689

4. Wooster R, Neuhausen SL, Mangion J, Quirk Y, Ford D, Collins N, Nguyen K, Seal S, Tran T, Averill D et al (1994) Localization of a breast cancer susceptibility gene, BRCA2, to chromosome 13q12-13. Science 265:2088-2090

5. Lynch HT, Silva E, Snyder C, Lynch JF (2008) Hereditary breast cancer: part I. Diagnosing hereditary breast cancer syndromes. Br J 14:3-13

6. King MC, Marks JH, Mandell JB (2003) Breast and ovarian cancer risks due to inherited mutations in BRCA1 and BRCA2. Science 302:643-646

7. Pal T, Permuth-Wey J, Betts JA, Krischer JP, Fiorica J, Arango H, LaPolla J, Hoffman M, Martino MA, Wakeley K, Wilbanks G, Nicosia S, Cantor A, Sutphen R (2005) BRCA1 and BRCA2 mutations account for a large proportion of ovarian carcinoma cases. Cancer 104:2807-2816

8. Bermejo-Perez MJ, Marquez-Calderon S, Llanos-Mendez A (2007) Effectiveness of preventive interventions in BRCA1/2 gene mutation carriers: a systematic review. Int $\mathrm{J}$ Cancer 121:225-231

9. Hacia JG, Brody LC, Chee MS, Fodor SP, Collins FS (1996) Detection of heterozygous mutations in BRCA1 using high density oligonucleotide arrays and two-colour fluorescence analysis. Nat Genet 14:441-447

10. Maitra A, Cohen Y, Gillespie SE, Mambo E, Fukushima N, Hoque MO, Shah N, Goggins M, Califano J, Sidransky D, Chakravarti A (2004) The human MitoChip: a high-throughput sequencing microarray for mitochondrial mutation detection. Genome Res 14:812-819

11. Fokstuen S, Lyle R, Munoz A, Gehrig C, Lerch R, Perrot A, Osterziel KJ, Geier C, Beghetti M, Mach F, Sztajzel J, Sigwart U, Antonarakis SE, Blouin JL (2008) A DNA resequencing array for pathogenic mutation detection in hypertrophic cardiomyopathy. Hum Mutat 29:879-885

12. Waldmüller S, Muller M, Rackebrandt K, Binner P, Poths S, Bonin M, Scheffold T (2008) Array-based resequencing assay for 
mutations causing hypertrophic cardiomyopathy. Clin Chem 54:682-687

13. Liu C, Aronow BJ, Jegga AG, Wang N, Miethke A, Mourya R, Bezerra JA (2007) Novel resequencing chip customized to diagnose mutations in patients with inherited syndromes of intrahepatic cholestasis. Gastroenterology 132:119-126

14. Mandal MN, Heckenlively JR, Burch T, Chen L, Vasireddy V, Koenekoop RK, Sieving PA, Ayyagari R (2005) Sequencing arrays for screening multiple genes associated with early-onset human retinal degenerations on a high-throughput platform. Invest Ophthalmol Vis Sci 46:3355-3362

15. Hacia JG (1999) Resequencing and mutational analysis using oligonucleotide microarrays. Nat Genet 21:42-47

16. Cowell JK, Hawthorn L (2007) The application of microarray technology to the analysis of the cancer genome. Curr Mol Med 7:103-120

17. Karaman MW, Groshen S, Lee CC, Pike BL, Hacia JG (2005) Comparisons of substitution, insertion and deletion probes for resequencing and mutational analysis using oligonucleotide microarrays. Nucleic Acids Res 33:e33

18. Cutler DJ, Zwick ME, Carrasquillo MM, Yohn CT, Tobin KP, Kashuk C, Mathews DJ, Shah NA, Eichler EE, Warrington JA, Chakravarti A (2001) High-throughput variation detection and genotyping using microarrays. Genome Res 11:1913-1925

19. Bunyan DJ, Eccles DM, Sillibourne J, Wilkins E, Thomas NS, Shea-Simonds J, Duncan PJ, Curtis CE, Robinson DO, Harvey JF, Cross NC (2004) Dosage analysis of cancer predisposition genes by multiplex ligation-dependent probe amplification. $\mathrm{Br} \mathbf{J}$ Cancer 91:1155-1159

20. Easton DF, Deffenbaugh AM, Pruss D, Frye C, Wenstrup RJ, Allen-Brady K, Tavtigian SV, Monteiro AN, Iversen ES, Couch FJ, Goldgar DE (2007) A systematic genetic assessment of 1, 433 sequence variants of unknown clinical significance in the BRCA1 and BRCA2 breast cancer-predisposition genes. Am J Hum Genet 81:873-883

21. Lee E, McKean-Cowdin R, Ma H, Chen Z, Van Den Berg D, Henderson BE, Bernstein L, Ursin G (2008) Evaluation of unclassified variants in the breast cancer susceptibility genes BRCA1 and BRCA2 using five methods: results from a population-based study of young breast cancer patients. Br Cancer Res 10:R19

22. Spearman AD, Sweet K, Zhou XP, McLennan J, Couch FJ, Toland AE (2008) Clinically applicable models to characterize BRCA1 and BRCA2 variants of uncertain significance. J Clin Oncol 26:5393-5400

23. Shendure J, Ji H (2008) Next-generation DNA sequencing. Nat Biotechnol 26:1135-1145

24. Silva E, Gatalica Z, Snyder C, Vranic S, Lynch JF, Lynch HT (2008) Hereditary breast cancer: part II. Management of hereditary breast cancer: implications of molecular genetics and pathology. Br J 14:14-24

25. Campeau PM, Foulkes WD, Tischkowitz MD (2008) Hereditary breast cancer: new genetic developments, new therapeutic avenues. Hum Genet 124:31-42 